DOI: $10.3901 / J M E .2021 .20 .172$

\title{
强风雨环境下高速列车运行安全特性*
}

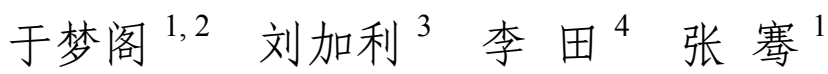 \\ (1. 青岛大学机电工程学院 青岛 266071; \\ 2. 青岛大学系统科学博士后流动站 青岛 266071; \\ 3. 中车青岛四方机车车辆股份有限公司 青岛 266111; \\ 4. 西南交通大学牵引动力国家重点实验室 成都 610031)
}

\begin{abstract}
摘要: 为确保高速列车在强风雨环境下安全运行, 结合 EULER-LAGRANGE 方法和计算多体动力学方法, 系统地研究风雨 环境下高速列车的气动特性及运行安全特性。基于非球形雨滴, 建立高速列车空气动力学计算模型, 并验证计算模型的准确 性, 进而计算强风雨环境下作用于高速列车的气动载荷。建立高速列车车辆系统动力学模型, 计算强风雨载荷作用下的高速 列车运行安全特性。研究结果表明, 在不同风速下, 高速列车的侧力、升力、侧滚力矩及摇头力矩均随降雨强度的增加而增 大, 且与降雨强度近似成线性关系, 对于点头力矩, 当风速较小时, 点头力矩随降雨强度的增加而增大, 而当风速较大时, 点头力矩随降雨强度的增加而减小。与单纯的强风环境相比, 降雨使得高速列车的运行安全特性进一步恶化, 在不同风速下, 高速列车脱轨系数、轮重减载率、倾覆系数及轮轴横向力均随降雨强度的增加而增大, 特别是当风速接近于临界风速时, 降 雨对高速列车运行安全特性的影响显著。当降雨强度为 $500 \mathrm{~mm} / \mathrm{h}$ 时, 由不同运行安全指标确定的高速列车安全运行的临界 风速降低 $2.3 \sim 4.2 \mathrm{~m} / \mathrm{s}$ 。研究结果可为高速列车在风雨环境下的安全限速提供参考。
\end{abstract}

关键词: 高速列车; 降雨强度; 气动性能; 运行安全; 临界风速

中图分类号: U270

\section{Operational Safety Characteristics of High-speed Train under Strong Wind and Rainfall Environment}

\author{
YU Mengge ${ }^{1,2}$ LIU Jiali $^{3}$ LI Tian $^{4}$ ZHANG Qian ${ }^{1}$ \\ (1. College of Mechanical and Electrical Engineering, Qingdao University, Qingdao 266071; \\ 2. Postdoctoral Research Station of System Science, Qingdao University, Qingdao 266071; \\ 3. CRRC Qingdao Sifang Co., Ltd., Qingdao 266111; \\ 4. State Key Laboratory of Traction Power, Southwest Jiaotong University, Chengdu 610031)
}

\begin{abstract}
In order to ensure the safe operation of the high-speed train exposed to strong wind and rain environment, the aerodynamic characteristics and operational safety characteristics of the high-speed train in wind and rain environment are systematically studied using the EULER-LAGRANGE method and computational multi-body dynamics method. The aerodynamic computational model of a high-speed train is set up based on non-spherical raindrops. After the the accuracy of the computational model is verified, the aerodynamic loads of the high-speed train operating in a strong wind and rain environment are computed. The vehicle system dynamic model of the high-speed train is established, and the operational safety characteristics of the high-speed train exposed to strong wind and rain loads are computed. The results show that the side force, lift force, rolling moment and yaw moment of the high-speed train increase with the increase of the rainfall intensity for a variety of wind speeds, and there is approximately a linear relationship between the aerodynamic loads and the rainfall intensity. For the pitch moment, it increases with the increase of the rainfall intensity
\end{abstract}

\footnotetext{
* 国家自然科学基金(51705267)、中国博士后科学基金(2018M630750)和牵 引动力国家重点实验室开放课题(TPL2005)资助项目。20201021 收到初 稿, 20210918 收到修改稿
} 
for the relatively smaller wind speeds, and decreases with the increase of the rainfall intensity for the relatively larger wind speed. Compared with the pure strong wind environment, the rainfall further deteriorates the operational safety characteristics of the high-speed train. The derailment coefficient, wheel load reduction rate, overturning coefficient, and axle lateral force of the high-speed train increase with the increase of the rainfall intensity for a variety of wind speeds. Especially, when the wind speed is close to the critical wind speed, the impact of rainfall on the operational safety characteristics of the high-speed train is significant. When the rainfall intensity is $500 \mathrm{~mm} / \mathrm{h}$, the critical wind speeds for the safe operation of the high-speed train determined by different operational safety indicators are reduced by $2.3-4.2 \mathrm{~m} / \mathrm{s}$. The results can provide a reference for the speed limit of the high-speed train in wind and rain environment.

Key words: high-speed train; rainfall intensity; aerodynamic performance; operational safety; critical wind speed

\section{0 前言}

近年来, 我国高速铁路发展迅速。国家 2016 年发布的《中长期铁路网规划》, 提出了构建 “八纵 八横” 高速铁路网。截至目前, 我国高速铁路里程 已达到 3.5 万 $\mathrm{km}$, 到 2030 年, 我国高速铁路里程 预计达到 4.5 万 $\mathrm{km}$, 我国已经成为世界上高速铁路 里程最长的国家。我国幅员辽阔, 气候环境多变, 当高速列车遭遇诸如强侧风、强降雨等恶劣天气, 作用于列车上的气动载荷显著增大, 严重威胁着高 速列车的运行安全。

有关强侧风环境下高速列车的气动特性和运 行安全特性, 国内外学者已经做了大量的研究工 作。BAKER ${ }^{[1]}$ 对侧风环境下铁路车辆运行安全分 析所涉及的列车模型、气动力计算方法、车辆系 统动力学模型及运行安全评估模型等进行了系统 地分析与探讨。DORIGATTI 等 ${ }^{[2]}$ 分别采用固定模 型试验和移动模型试验对比分析了横风下高速列 车的压力分布及气动载荷特性。LI 等 ${ }^{[3]}$ 采用六种 不同的湍流模型研究了高速列车在横风下的流场 特性、表面压力和气动力特性。郗艳红等 ${ }^{[4]}$ 建立 了头车-中间车-尾车三编组的高速列车模型, 采 用分离浴方法研究了横风环境下高速列车的非定 常气动特性, 指出各节车的非定常气动载荷的均 值与定常流动计算结果基本一致, 且三节车中头 车的横风气动性能最恶劣。除了自然风之外, 降 雨也是非常常见的一种气候条件。铁路网密集规 划的东南沿海地带, 处于季风区, 年降水量在 $1600 \mathrm{~mm}$ 以上。相对于单纯的侧风环境, 风雨耦 合使得列车周围流场更加复杂，气动效应加剧。 因此, 非常有必要研究风雨耦合作用下高速列车 的气动性能和运行安全特性。

目前, 有关风雨环境对高速列车气动特性及 运行安全特性的影响分析, 已经有一些初步的研
究成果。由于风雨环境下高速列车气动性能实车 试验和风洞试验开展比较困难, 尚未有相关研究 报导, 目前的研究以数值仿真为主。风雨环境属 于气-液两相流流动, 常用的数值模拟方法包括 EULER-LAGRANGE 方法和 EULER-EULER 方 法。NESTOR ${ }^{[5]}$ 、敬俊娥等 ${ }^{[6]}$ 、岳显斐等 ${ }^{[7]}$ 采用 EULER-LAGRANGE 方法研究了风雨联合作用下 降雨强度对列车气动载荷的影响, 提出与无雨环 境相比, 降雨会使得列车的气动载荷增大。倪守 隆 $^{[8]}$ 采用 EULER-LAGRANGE 方法和多体系统动 力学方法研究了强风雨环境下高速列车的运行安 全特性, 并给出降雨强度为 $60 \mathrm{~mm} / \mathrm{h}$ 时, 列车安 全运行的车速-风速关系曲线。SHAO 等 ${ }^{[9]}$ 采用 EULER-EULER 方法研究了风雨耦合对高速列车 气动特性的影响, 并采用准静力矩平衡方法给出 了高速列车在 $60 \mathrm{~mm} / \mathrm{h}$ 降雨强度下的临界风速。 由于多相流模拟计算量巨大, 目前的研究大多简 化了转向架、风挡等细部结构。转向架会对列车 底部流场产生影响, 进而影响到风雨环境下高速 列车气动特性评估的准确性。于梦阁等 ${ }^{[10]}$ 考虑了 转向架与风挡等细部结构, 研究了 $70 \mathrm{~m} / \mathrm{s}$ 合成风 速下侧偏角和降雨强度对气动特性的影响, 给出 了气动载荷系数的拟合函数。但目前的工作在模 拟降雨环境时, 均假设雨滴是球形颗粒。实际观 测表明: 当雨滴粒径较小, 雨滴形状接近球形, 当雨滴粒径较大时, 在表面张力、静水压力和空 气动力压力的共同作用下, 雨滴变为纺锤形, 球 形雨滴假设与实际相差较大。此外, 目前的工作 主要集中在降雨对列车周围流场及气动特性的影 响, 较少考虑降雨对列车运行安全特性的影响。

为此, 本文将雨滴考虑为非球形颗粒, 利用 EULER-LAGRANGE 方法建立强风雨环境下高速 列车空气动力学计算模型, 并建立高速列车车辆系 统动力学模型, 研究强风雨环境对高速列车气动性 能及运行安全特性的影响。 


\section{1 计算方法}

强风雨环境本质上为气液两相流流动, 其数值 模拟包括连续相空气的模拟和离散相雨滴的模拟。 由于雨滴在空气中的浓度较低(远低于 $10 \%$ ), 本文 采用 EULER-LAGRANGE 方法对风雨环境进行模 拟。对于 EULER 流体部分, 利用 NAVIER-STOKES 方程求解列车周围气流流动问题, 湍流模型采用 SST $k-\omega$ 模型。对离散相颗粒部分, 其 LAGRANGE 运动方程为 ${ }^{[11]}$

$$
\frac{\mathrm{d} u_{\mathrm{p} i}}{\mathrm{~d} t}=\frac{18 \mu}{\rho_{\mathrm{p}} d_{\mathrm{p}}^{2}} \frac{C_{\mathrm{D}_{\mathrm{p}}} R e_{\mathrm{p}}}{24}\left(u_{i}-u_{\mathrm{p} i}\right)+\frac{g_{i}\left(\rho_{\mathrm{p}}-\rho\right)}{\rho_{\mathrm{p}}}
$$

式中, $u_{\mathrm{p} i}$ 为雨滴速度分量, $u_{i}$ 为空气速度分量, $t$ 为时间, $\mu$ 为动力黏度, $\rho_{\mathrm{p}}$ 为雨滴密度, $\rho$ 为空气密 度, $d_{\mathrm{p}}$ 为雨滴粒径, $C_{\mathrm{Dp}}$ 为曳力系数, $R e_{\mathrm{p}}$ 为相对 Reynolds 数, $g_{i}$ 为重力加速度分量。

相对 Reynolds 数的定义为

$$
R e_{\mathrm{p}}=\frac{\rho d_{\mathrm{p}}\left|u_{\mathrm{p} i}-u_{i}\right|}{\mu}
$$

曳力系数主要由颗粒形状决定。当降雨强度不 大, 雨滴粒径较小时, 雨滴形状接近球形, 曳力系 数的计算公式为 ${ }^{[12]}$

$$
C_{\mathrm{D}_{\mathrm{p}}}=a_{1}+\frac{a_{2}}{R e_{\mathrm{p}}}+\frac{a_{3}}{R e_{\mathrm{p}}^{2}}
$$

式中, $a_{1} 、 a_{2} 、 a_{3}$ 为经验系数。

然而, 随着雨滴粒径增大, 雨滴形状逐渐由球 体变为纺锤体, 雨滴的非球形特性显著。对于非球 形颗粒, 曳力系数的计算公式为 ${ }^{[13]}$

$$
\begin{gathered}
C_{\mathrm{Dp}}=\frac{24}{R e_{\mathrm{pe}}}\left(1+b_{1} \operatorname{Re}_{\mathrm{pe}}^{b_{2}}\right)+\frac{b_{3} R e_{\mathrm{pe}}}{b_{4}+R e_{\mathrm{pe}}} \\
b_{1}=\exp \left(2.3288-6.4581 \phi+2.4486 \phi^{2}\right) \\
b_{2}=0.0964+0.5565 \phi \\
b_{3}=\exp \left(4.905-13.8944 \phi+18.4222 \phi^{2}-10.2599 \phi^{3}\right) \\
b_{4}=\exp \left(1.4681+12.2584 \phi-20.7322 \phi^{2}+15.8855 \phi^{3}\right)
\end{gathered}
$$

式中, $\varphi$ 表示雨滴非球形度, $R e_{\mathrm{pe}}$ 为采用等效粒径 计算得到的 Reynolds 数, 等效粒径定义为与非球形 粒子体积相等的球体的直径。

雨滴非球形度的定义为 ${ }^{[13]}$

$$
\varphi=s / S
$$

式中, $s$ 为与非球形颗粒体积相等的球体的表面积, $S$ 为非球形粒子的实际表面积。非球形度的最大值 为 1 。

雨滴粒径主要由降雨强度决定。BEST 给出了 不同降雨强度下雨滴粒径的计算公式 ${ }^{[14]}$

$$
d_{\mathrm{p}}=A I^{m}\left(\frac{n-1}{n}\right)^{1 / n}
$$

式中, $I$ 表示降雨强度, $A 、 n 、 m$ 均为经验系数, $A=1.30, n=2.25, m=0.232$ 。

雨滴下落过程中, 当周围空气作用力与自身重 力相平衡时, 雨滴将匀速下降, 此时的降落速度称 为雨滴降落末速度 ${ }^{[15]}$

$$
V_{\mathrm{p}}=9.58\left(1-\exp \left(-\left(\frac{10^{3} d_{\mathrm{p}}}{1.77}\right)^{1.147}\right)\right)
$$

采用 EULER-LAGRANGE 方法进行多相流模 拟时，一般有两种模拟方法：一种只考虑连续相对 离散相的影响, 而不考虑离散相对连续相的影响; 另外一种考虑连续相和离散相的相互影响, 称为相 间耦合。本文采用相间耦合方法模拟强风雨环境, 雨滴对空气的影响是通过相间动量交换实现，将雨 滴的动量源项引入到空气动量方程中, 项间动量交 换为 ${ }^{[11]}$

$$
M_{i}=\sum \frac{18 \mu}{\rho_{\mathrm{p}} d_{\mathrm{p}}^{2}} \frac{C_{\mathrm{D}_{\mathrm{p}}} R e_{\mathrm{p}}}{24}\left(u_{i}-u_{\mathrm{p} i}\right) \dot{m}_{p} \Delta t
$$

式中, $M_{i}$ 表示相间动量交换分量, $\dot{m}_{p}$ 表示单位体 积内的颗粒质量流率; $\Delta t$ 表示作用时间。

\section{2 计算模型}

\section{1 非球形雨滴}

雨滴降落末速度是表征降雨特性的一个重要的 物理量。MARKOWITZ ${ }^{[15]}$ 对 BEST 的测量结果进行 分析, 给出雨滴降落末速度与雨滴等效粒径的关系, 即式(7)。通过开展不同雨滴粒径下的雨滴降落数值 模拟，获得不同粒径雨滴的降落末速度，并通过与 式(7)的对比, 验证 EULER-LAGRANGE 方法的可 靠性及球形雨滴的适用性。

利用计算流体力学 (Computational fluid dynamics, CFD)方法模拟雨滴的降落速度。计算区 域如图 1 所示, 计算区域的长度为 $10 \mathrm{~m}$, 宽度为 $2 \mathrm{~m}$, 高度为 $30 \mathrm{~m}$ 。对于连续相空气，计算区域前后端、 左右侧和顶端均设置为对称边界，底端设置为壁面 
边界; 对于离散相雨滴, 在计算区域顶端布置 9 个 入射点, 相邻入射点的间距为 $1 \mathrm{~m}$ 。首先将雨滴假 设为球形颗粒, 雨滴的粒径分别取 $0.5 \mathrm{~mm} 、 1.0 \mathrm{~mm}$ 、 $1.5 \mathrm{~mm} 、 2.0 \mathrm{~mm} 、 2.5 \mathrm{~mm} 、 3.0 \mathrm{~mm} 、 3.5 \mathrm{~mm} 、 4.0 \mathrm{~mm}$ 和 $4.5 \mathrm{~mm}$, 并由各个入射点进入计算区域, 其初始 降落速度均设为 $1 \mathrm{~m} / \mathrm{s}$ 。

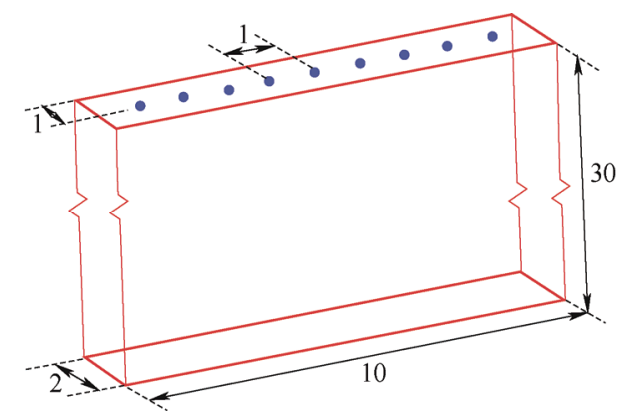

图 1 计算区域(m)

雨滴进入计算区域后, 在自身重力和空气阻力 的作用下运动, 最终匀速降落。图 2 给出不同雨滴 粒径下, 雨滴降落速度随降落时间的变化规律。由 图 2 可以看出, 开始时, 雨滴速度较小, 雨滴重力 大于空气阻力, 雨滴加速降落; 随着时间的增加, 雨滴降落速度逐渐增大, 并趋于稳定值, 此稳定 值即为雨滴降落末速度。雨滴降落末速度主要受 雨滴粒径的影响, 雨滴粒径越大, 降落末速度也 越大。

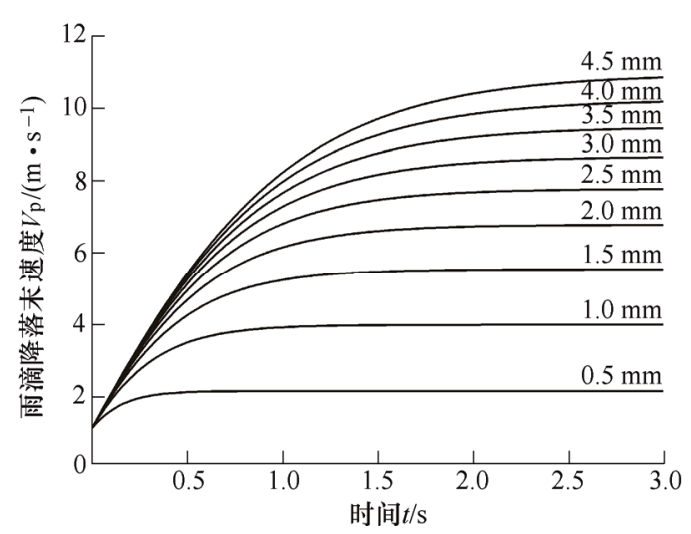

图 2 雨滴降落速度随时间的变化

图 3 给出球形雨滴假设下, 雨滴降落末速度的 数值计算结果与 MARKOWITZ 的结果对比。由图 3 可以看出, 当雨滴粒径较小时, 基于球形雨滴假设 的 CFD 数值计算结果与 MARKOWITZ 的结果相差 很小。然而, 随着雨滴粒径增大, 基于球形雨滴假 设的 CFD 数值计算结果与 MARKOWITZ 的结果差 异愈发明显, 当雨滴粒径为 $2.0 \mathrm{~mm}$ 时, CFD 数值 计算结果比 MARKOWITZ 的结果偏大 $3.0 \%$, 当雨 滴粒径为 $4.5 \mathrm{~mm}$ 时, CFD 数值计算结果比
MARKOWITZ 的结果偏大 $20.5 \%$ 。因此, 当降雨 强度比较大时, 基于球形雨滴假设的 CFD 模拟与实 际相差较大, 需要考虑雨滴的非球形特性。

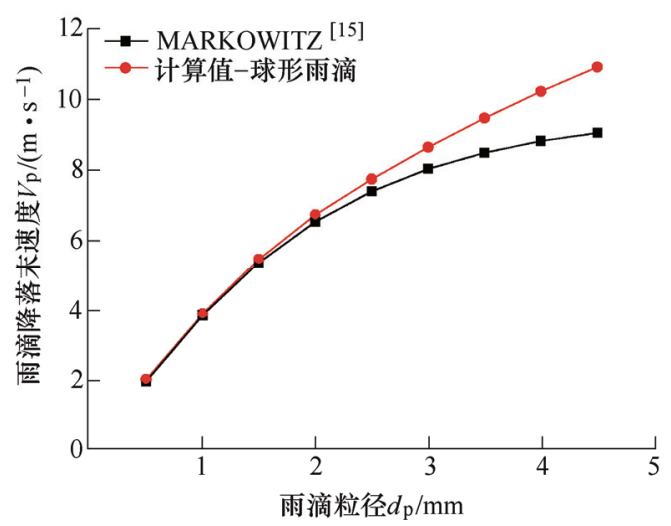

图 3 雨滴降落末速度-球形雨滴

对于非球形雨滴, CFD 数值模拟时, 在相同的 等效粒径下, 非球形度 $\varphi$ 代表雨滴形状偏离球形的 程度, 其取值直接影响电力系数, 进而影响雨滴降 落末速度的准确预测, 因此需要确定不同雨滴等效 粒径下非球形度的取值。通过研究不同雨滴等效粒 径下非球形度的取值与雨滴降落末速度的关系, 并 结合 MARKOWITZ 的结果, 建立不同雨滴等效粒 径下非球形度的计算公式 ${ }^{[16]}$

$$
\phi=0.0016 d_{\mathrm{pe}}^{2}-0.0277 d_{\mathrm{pe}}+1.0355
$$

式中, $d_{\mathrm{pe}}$ 为非球形雨滴的等效粒径, 其单位为 $\mathrm{mm}$, 公式的适用范围是 $2 \mathrm{~mm} \leqslant d_{\mathrm{pe}} \leqslant 4.5 \mathrm{~mm}$ 。

图 4 给出采用非球形雨滴(粒径小于 $2 \mathrm{~mm}$ 时采 用球形雨滴)时, 不同雨滴等效粒径下, 雨滴降落末 速度的计算值与 MARKOWITZ 的结果的比较。

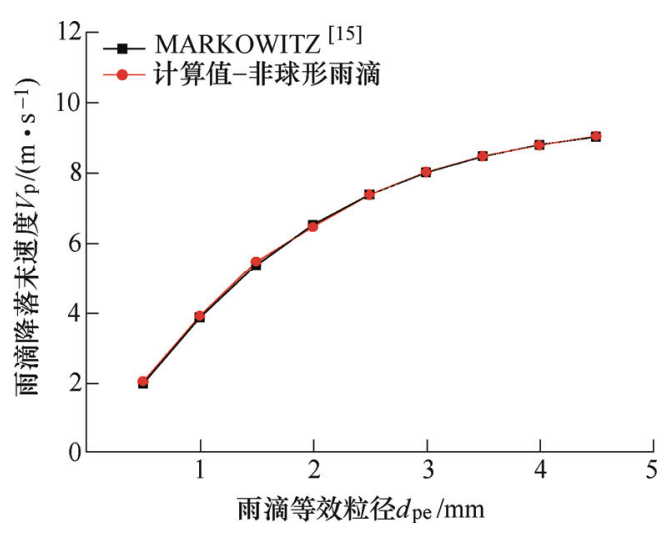

图 4 雨滴降落末速度-非球形雨滴

由图 4 可以看出, 不同雨滴粒径下, 雨滴降落 末速度计算值与 MARKOWITZ 的结果相差很小, 差异在 $1 \%$ 以内。因此, 非球形雨滴假设可以更好地 适用于大粒径雨滴的数值模拟。 


\section{2 列车空气动力学模型}

参考国内某高速列车，建立高速列车三维几 何外形。与单相流流场相比, 高速列车多相流流 场模拟更加复杂，计算量也更大，同时考虑到高 速列车头车的横风运行安全性最差 ${ }^{[4,17-19]}$, 本文 将重点关注头车的气动性能及运行安全特性。为 此, 本文建立了如图 5 所示的高速列车模型, 此 高速列车模型由“头车十流线型车尾”构成, 流线型 车头和车尾的形状完全相同, 其长度为 $9.1 \mathrm{~m}$, 并 考虑了转向架、风挡等细部结构。高速列车头车 车身长度为 $25.45 \mathrm{~m}$, 宽度为 $3.38 \mathrm{~m}$, 高度为 $3.7 \mathrm{~m}$, 风挡宽度 $0.5 \mathrm{~m}$ 。

高速列车风雨多相流流场数值模拟时, 雨滴 由计算区域的顶端进入计算区域, 受列车前方及 侧方来流的影响, 雨滴将会产生水平运动速度, 其大小接近于入口风速大小 ${ }^{[20]}$ 。因此, 为了保证 从计算区域顶端入射的雨滴能完全降落到列车表 面, 列车前端及侧面迎风侧的计算区域应该足够 长。本文中, 列车速度为 $300 \mathrm{~km} / \mathrm{h}$, 风速为 $10 \sim$ $30 \mathrm{~m} / \mathrm{s}$, 降雨强度为 $0 \sim 500 \mathrm{~mm} / \mathrm{h}$, 根据入口风速 与雨滴降落末速度可以确定列车前端及侧面迎风 侧的距离要求。

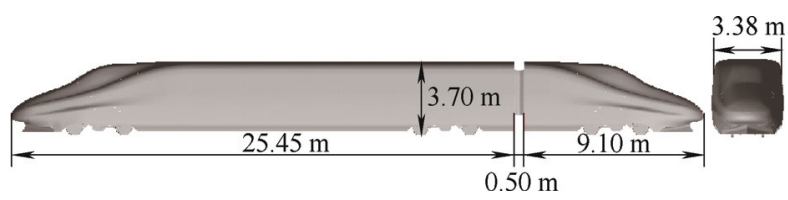

图 5 高速列车模型

高速列车空气动力学计算区域如图 6 所示。计 算区域的长度为 $900 \mathrm{~m}$, 宽度为 $300 \mathrm{~m}$, 高度为 $30 \mathrm{~m}$, 前端速度入口到列车头部鼻雉的距离为 $660 \mathrm{~m}$, 侧 面速度入口到列车纵向对称面的距离为 $200 \mathrm{~m}$, 此 距离可保证由计算区域顶端进入的雨滴能够完全降 落到列车表面。连续相空气及离散相雨滴的边界条 件设置如表 1 所示, 区域前后端及左右侧以面向列 车的前进方向进行定义。

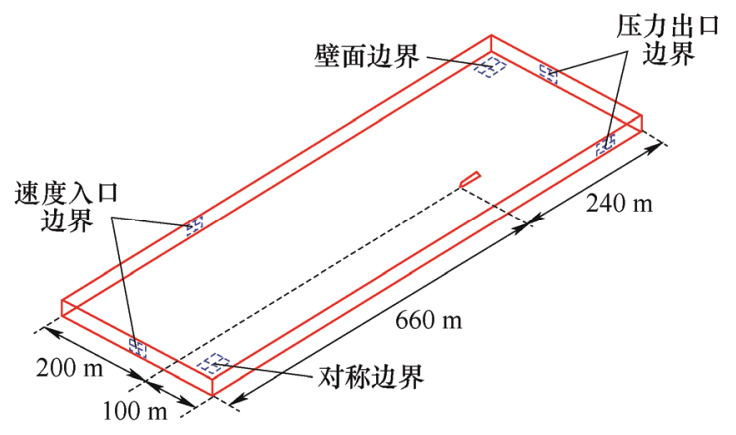

图 6 计算区域
表 1 边界条件

\begin{tabular}{ccc}
\hline 边界 & 连续相-空气 & 离散相-雨滴 \\
\hline 区域前端 & Velocity-inlet & escape \\
区域后端 & Pressure outlet & escape \\
区域右侧 & Velocity-inlet & escape \\
区域左侧 & Pressure outlet & escape \\
区域顶端 & Symmetry & - \\
区域底端 & wall & trap \\
列车 & wall & wall-film \\
\hline
\end{tabular}

网格划分时，面网格采用三角形网格，体网格 采用四面体网格。为验证网格精度, 本文划分了两 套网格, 网格尺寸参数如表 2 所示。车体、转向架 及风挡表面均设置边界层, 边界层网格设置相同, 边界层的层数为 12 , 增长比为 1.2 , 第一层的网格 厚度为 $0.01 \mathrm{~mm}$, 使得列车表面 $y^{+}$在 1 附近，以满 足 SST $k-\omega$ 湍流模型的计算要求。图 7 给出高速列 车头型网格、转向架网格及边界层网格示意图。

表 2 网格设置

\begin{tabular}{ccccc}
\hline 网格 & 车体 $/ \mathrm{mm}$ & 转向架和风挡 $/ \mathrm{mm}$ & 区域边界 $/ \mathrm{mm}$ & 网格量 $/$ 万 \\
\hline 网格 1 & 60 & 30 & 2400 & 1700 \\
网格 2 & 50 & 25 & 2000 & 2500 \\
\hline & & & \\
& & & \\
& & & \\
& & &
\end{tabular}

(a) 列车表面网格

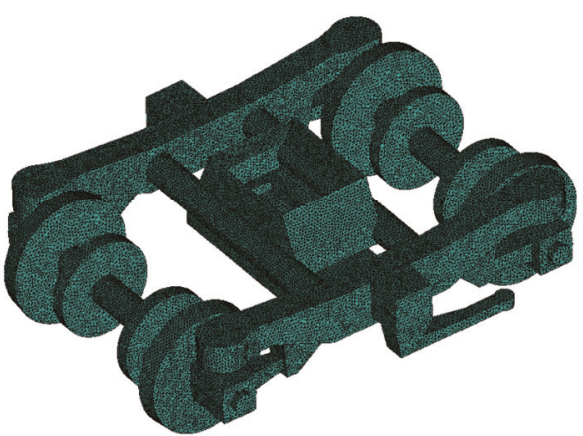

(b) 转向架网格

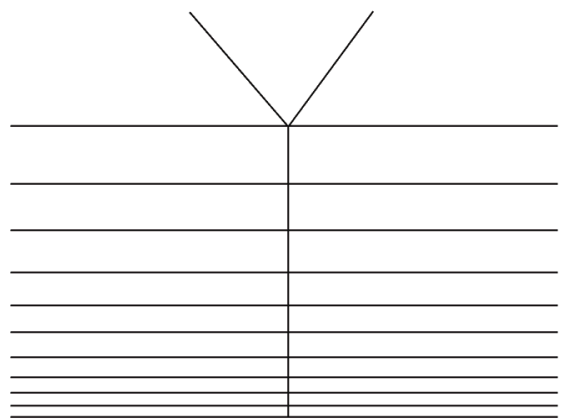

(c) 边界层网格

图 7 计算网格 
采用 Fluent 软件开展列车空气动力学计算。为 了验证本文的高速列车空气动力学计算模型的准确 性, 开展不同侧偏角下的高速列车空气动力学性能 计算, 并与既有的风洞试验结果 ${ }^{[21]}$ 进行对比。图 8 给出了不同侧偏角下高速列车侧力系数计算值与试 验值的对比。由图 8 可以看出, 不同侧偏角下, 高 速列车侧力系数的计算值与试验值的差异均较小, 误差在 $5 \%$ 以内, 可以满足工程计算精度要求。同时, 网格 1 和网格 2 的计算结果基本相同, 其差异在 1\% 以内。因此, 本文采用网格 1 进行高速列车空气动 力学计算。

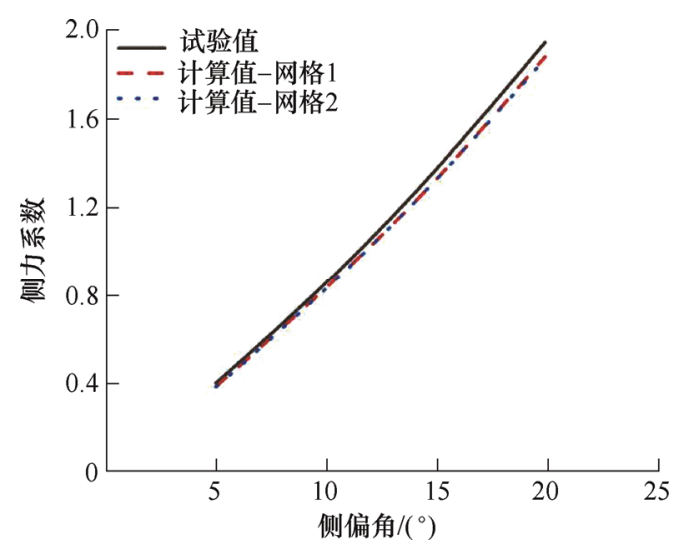

图 8 气动侧力系数对比

\section{3 车辆系统动力学模型}

利用 SIMPACK 软件建立高速列车头车车辆系 统动力学模型(图 9)。车辆系统动力学模型主要由车 辆动力学模型和轮轨关系构成。车辆动力学模型包 括车体、构架、轮对和转臂等, 其中每个部件均假 设为刚体, 不考虑其弹性变形。车体、构架和轮对 均有 6 个自由度, 即纵向、横向、垂向、侧滚、摇 头和点头, 转臂有 1 个自由度, 即点头。整个车辆 动力学模型共有 50 个自由度。轮对和构架之间采用 一系悬挂系统, 包括转臂节点、轴箱弹簧和垂向减 振器等, 车体和构架之间采用二系悬挂系统, 包括 空气弹簧、抗蛇形减振器、横向止挡和牵引拉杆等。

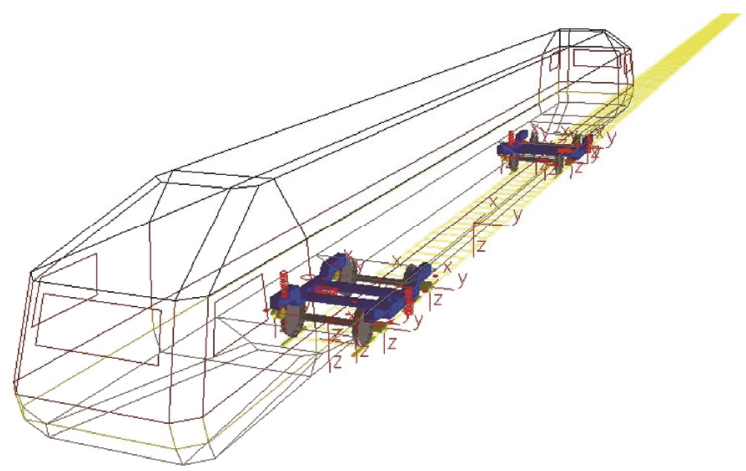

图 9 车辆系统动力学模型
车辆系统的悬挂部件具有非线性特性, 本文采用力 元对悬挂部件建模, 考虑了抗蛇形减振器和一系垂 向减振器的非线性阻尼特性及横向止挡的非线性刚 度特性 ${ }^{[22-23]}$ 。轮轨关系是高速列车车辆系统动力学 模型的重要组成部分。本文中, 车轮踏面采用 LMA 磨耗型踏面, 钢轨采用 T60 钢轨, 采用 Kalker 法则计 算轮轨蠕滑力。轨道不平顺激励采用国内某高速铁 路实测轨道不平顺。

在高速列车车辆系统动力学模型建立之后, 将 强风雨气动载荷作为激励载荷作用于车辆系统动力 学模型中, 评估强风雨环境下高速列车的运行安全 特性。由于各个部件均视作刚体, 本文将作用于列 车表面的分布压力向车体某一点简化, 得到作用于 车体某一点的集中载荷, 并将此集中载荷作为外加 载荷施加于车辆系统动力学模型的同一位置作用 点。高速列车运行安全指标包括脱轨系数、轮重减 载率、倾覆系数及轮轴横向力等, 其限值分别如下: 脱轨系数不大于 0.8 ; 动态轮重减载率不大于 0.8 ; 倾覆系数不大于 0.80 ; 轮轴横向力不大于 $10+P_{0} / 3$, 其中, $P_{0}$ 为轴载荷。

\section{3 计算结果分析}

数值计算时, 车速取 $300 \mathrm{~km} / \mathrm{h}$, 风速分别取 $10 \mathrm{~m} / \mathrm{s} 、 15 \mathrm{~m} / \mathrm{s} 、 20 \mathrm{~m} / \mathrm{s} 、 25 \mathrm{~m} / \mathrm{s} 、 30 \mathrm{~m} / \mathrm{s}$, 降雨强度 分别取 $0 \mathrm{~mm} / \mathrm{h} 、 100 \mathrm{~mm} / \mathrm{h} 、 200 \mathrm{~mm} / \mathrm{h} 、 300 \mathrm{~mm} / \mathrm{h}$ 、 $400 \mathrm{~mm} / \mathrm{h} 、 500 \mathrm{~mm} / \mathrm{h}$ 。

图 10 给出车速为 $300 \mathrm{~km} / \mathrm{h}$, 风速为 $25 \mathrm{~m} / \mathrm{s}$, 降 雨强度分别为 $0 \mathrm{~mm} / \mathrm{h}$ 和 $500 \mathrm{~mm} / \mathrm{h}$ 时, 高速列车的 流线型头型压力分布云图。由图 10 可以看出, 在强 风雨环境下, 由于雨滴的冲击作用, 流线型头型迎 风侧区域的正压较单纯的强风环境有所增大, 流线 型头型背风侧区域的负压也有所增大, 由此将导致 车辆侧向力增大, 列车横向气动性能更为恶劣。

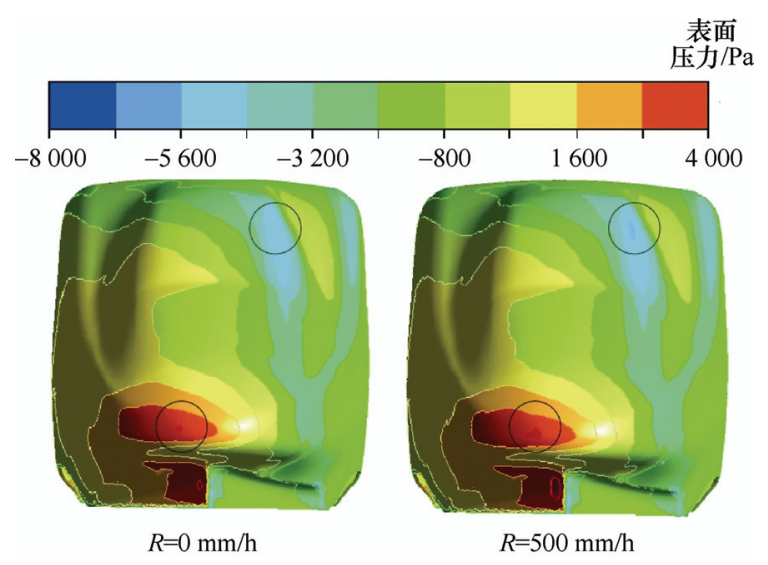

图 10 流线型头型表面压力分布 
图 11 给出车速 $300 \mathrm{~km} / \mathrm{h}$ 时, 高速列车的侧力、 升力、侧滚力矩、摇头力矩及点头力矩随降雨强度 和风速的变化规律。由图 11 可以看出, 对于固定的 车速和风速, 高速列车的侧力、升力、侧滚力矩及 摇头力矩均随着降雨强度的增加而增大, 且与降雨 强度近似成线性关系。对于点头力矩, 在车速 $300 \mathrm{~km} / \mathrm{h}$ 时, 高速列车的点头力矩随着风速的增加 先增大后减小; 当风速较小时, 高速列车的点头力 矩与风速正相关, 点头力矩随着降雨强度的增加而 增大; 而当风速较大时, 高速列车的点头力矩与风 速负相关, 点头力矩随着降雨强度的增加而减小。 综合而言, 与单纯的强风环境相比, 降雨使得高速 列车的气动性能进一步恶化。

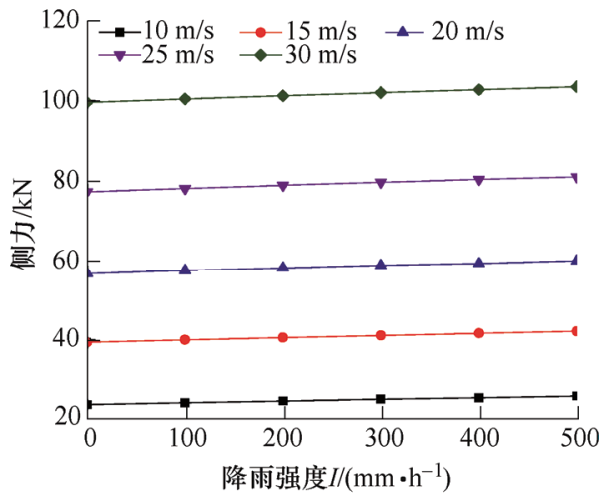

(a) 侧力

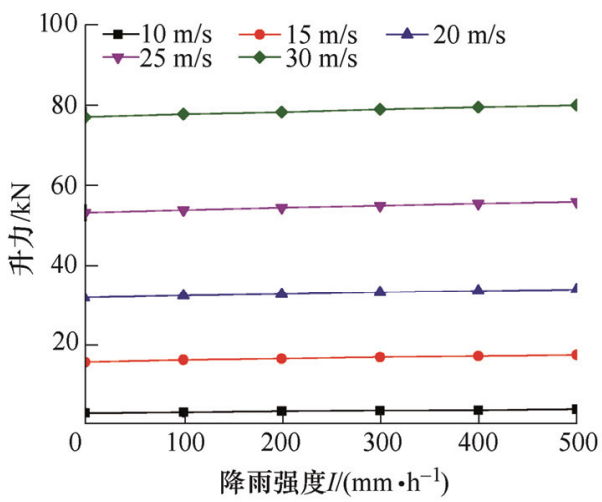

(b) 升力

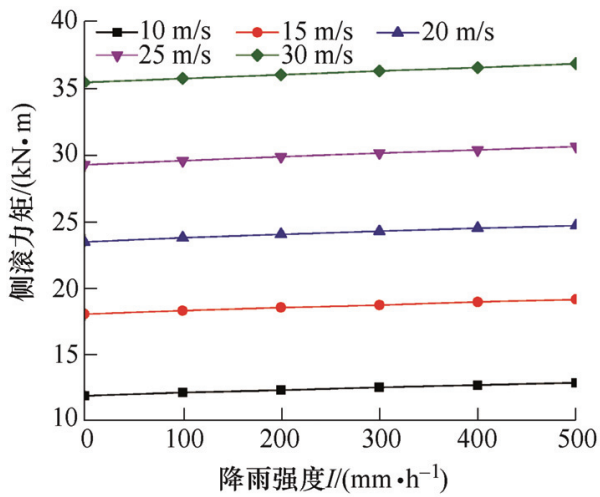

(c) 侧滚力矩

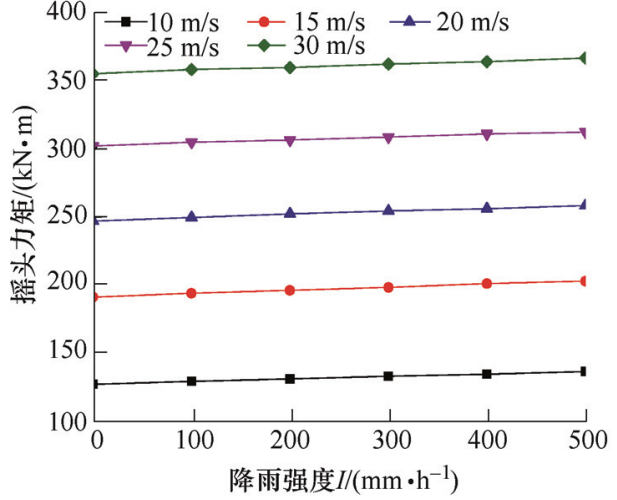

(d) 摇头力矩

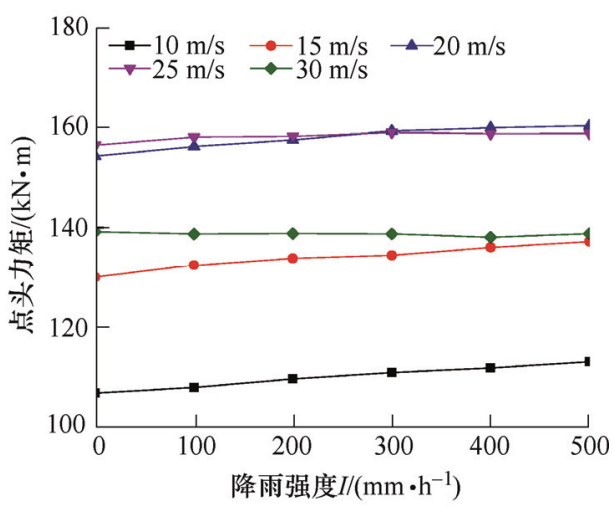

(e) 点头力矩

图 11 气动载荷变化规律

强风雨环境对高速列车运行安全特性的影响, 最终体现在运行安全指标的变化。图 12 给出车速 $300 \mathrm{~km} / \mathrm{h}$ 时, 高速列车的脱轨系数、轮重减载率、 倾覆系数及轮轴横向力随降雨强度和风速的变化规 律, 图中的水平虚线表示各个运行安全指标的限值。

由图 12 可以看出, 对于相同的车速和风速, 高 速列车的运行安全指标随着降雨强度的增加而增 大; 与单纯的强风环境相比, 降雨使得高速列车的 运行安全特性进一步恶化。需要特别注意的是，当 风速较小时, 降雨强度对高速列车运行安全特性的 影响较小; 当风速达到 $25 \mathrm{~m} / \mathrm{s}$ 时, 降雨强度对高速 列车运行安全特性的影响变得非常显著, 这主要是 因为在 $25 \mathrm{~m} / \mathrm{s}$ 的风速下, 高速列车的运行安全特性 较差, 轮重减载率及轮轴横向力已接近安全限值, 此时高速列车车辆系统的非线性动力响应已非常显 著。在这种情况下, 运行安全指标对外加气动载荷 (如风载荷或雨载荷)的变化特别灵敏, 由降雨引起 的气动载荷增量会对高速列车的运行安全特性产生 显著影响，表现为曲线簇分叉。而当风速增大到 30 $\mathrm{m} / \mathrm{s}$, 运行安全指标远超出限值, 此时, 运行安全指 标对外加气动载荷变化的灵敏度降低, 由降雨引起 的气动载荷增量依然会对高速列车的运行安全特性 产生影响, 只是差异没有风速 $25 \mathrm{~m} / \mathrm{s}$ 时显著。由此 
可知, 在某一固定车速下, 当风速接近于临界风速

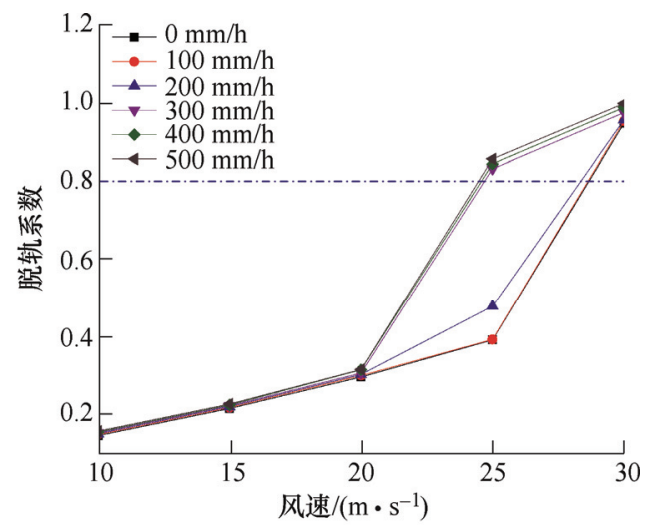

(a) 脱轨系数

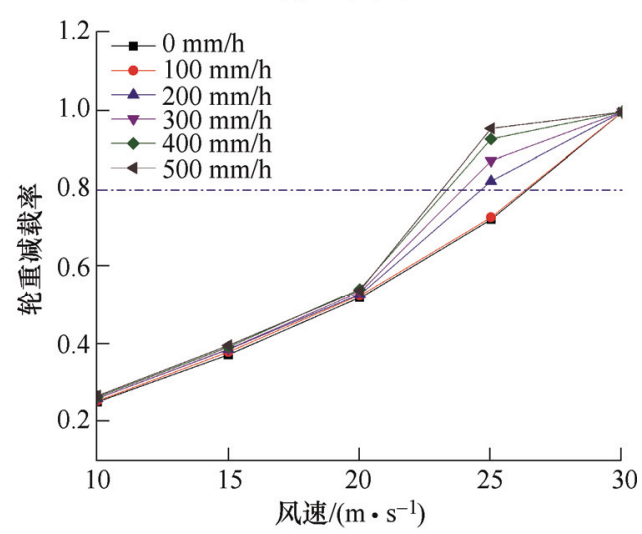

(b) 轮重减载率

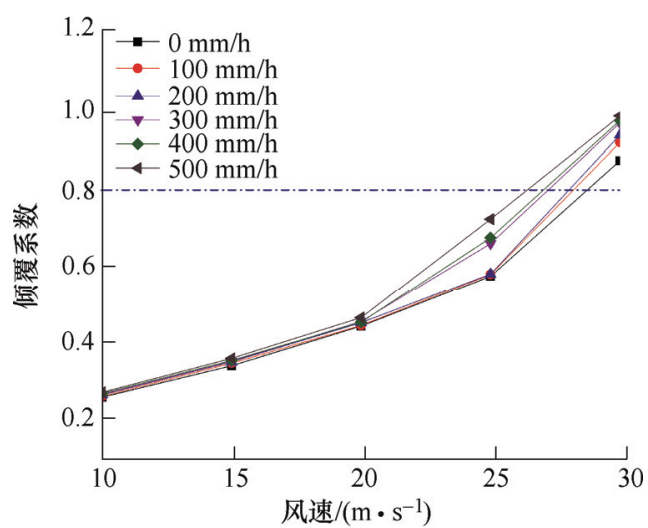

(c) 倾覆系数

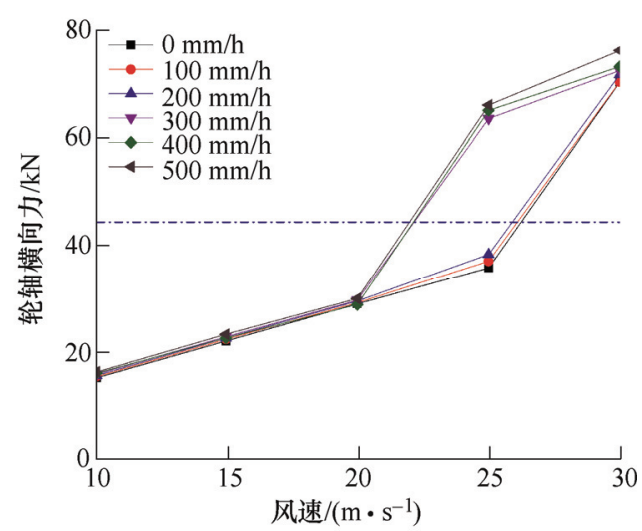

(d) 轮轴横向力

图 12 运行安全指标变化规律
时, 气动载荷的变化对列车运行安全的影响变得显 著, 此时降雨引起的气动载荷增量会使得列车的安 全特性迅速恶化。对于不同的车速, 临界风速取值 也不同。

根据图 12 中高速列车各运行安全指标的变化 规律及其限值, 可以确定出高速列车以 $300 \mathrm{~km} / \mathrm{h}$ 安 全运行时的临界风速, 如图 13 所示。

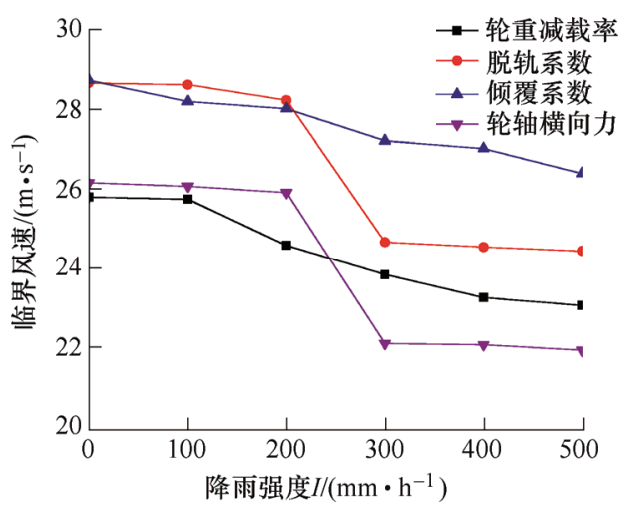

图 13 临界风速曲线

由图 13 可以看出, 与单纯的强风环境相比, 强 风雨环境下高速列车的运行安全性变差, 高速列车 安全运行的临界风速进一步降低。与单纯的强风环 境相比, 当降雨强度为 $500 \mathrm{~mm} / \mathrm{h}$ 时, 由脱轨系数确 定的临界风速降低 $4.2 \mathrm{~m} / \mathrm{s}$, 由轮重减载率确定的临 界风速降低 $2.7 \mathrm{~m} / \mathrm{s}$, 由倾覆系数确定的临界风速降 低 $2.3 \mathrm{~m} / \mathrm{s}$, 由轮轴横向力确定的临界风速降低 $4.2 \mathrm{~m} / \mathrm{s}$ 。

\section{4 结论}

(1) 当雨滴粒径较小时, 可以采用球形雨滴假 设, 但当雨滴粒径大于 $2 \mathrm{~mm}$ 时, 则需要考虑雨滴 的非球形特性。

(2) 不同风速下, 高速列车的侧力、升力、侧 滚力矩及摇头力矩均随降雨强度的增加而增大, 且 与降雨强度近似成线性关系。对于点头力矩, 当风 速较小时, 点头力矩随降雨强度的增加而增大, 而 当风速较大时, 点头力矩随降雨强度的增加而减小。

(3) 不同风速下, 高速列车的脱轨系数、轮重 减载率、倾覆系数及轮轴横向力均随降雨强度的增 加而增大, 特别是当风速接近于临界风速时, 降雨 对高速列车运行安全特性的影响非常显著。

(4) 与单纯的强风环境相比, 降雨使得高速列 车的运行安全性进一步恶化。在车速 $300 \mathrm{~km} / \mathrm{h}$, 降 雨强度 $500 \mathrm{~mm} / \mathrm{h}$ 时, 由不同运行安全指标确定的高 速列车安全运行的临界风速降低 $2.3 \sim 4.2 \mathrm{~m} / \mathrm{s}$ 。 


\section{参 考 文 献}

[1] BAKER C. A framework for the consideration of the effects of crosswinds on trains[J]. Journal of Wind Engineering and Industrial Aerodynamics, 2013, 123: 130-142.

[2] DORIGATTI F, STERLING M, BAKER C J, et al. Crosswind effects on the stability of a model passenger train - a comparison of static and moving experiments[J]. Journal of Wind Engineering and Industrial Aerodynamics, 2015, 138: 36-51.

[3] LI T, QIN D, ZHANG J Y. Effect of RANS turbulence model on aerodynamic behavior of trains in crosswind[J]. Chinese Journal of Mechanical Engineering, 2019， 32: 85.

[4] 郗艳红, 毛军, 高亮, 等. 基于分离涡方法的高速列车 横风非定常气动特性[J]. 中南大学学报, 2015，46(3): 1129-1139.

XI Yanhong, MAO Jun, GAO Liang, et al. Aerodynamic force/moment for high-speed train in crosswind field based on DES[J]. Journal of Central South University, 2015, 46(3): 1129-1139.

[5] NESTOR D M. 横风大雨对高速列车绕流的影响 [D]. 北京: 北京交通大学，2013.

NESTOR D M. Influence of the crosswind and heavy rain on the flow around a high-speed train[D]. Beijing: Beijing Jiaotong University, 2013.

[6] 敬俊娥, 高广军. 风雨联合作用下高速列车受力数值模 拟[J]. 铁道科学与工程学报, 2013, 10(3): 99-102.

JING June, GAO Guangjun. Simulation of the action effect of wind-drive rain on high-speed train[J]. Journal of Railway Science and Engineering, 2013，10(3): 99-102.

[7] 岳显斐, 曾秋兰, 李振山, 等. 挟雨风对高速列车气动 特性及运行稳定性影响的数值模拟 [J]. 中国沙漠, 2016, 36(4): 943-950.

YUE Yufei, ZENG Qiulan, LI Zhenshan, et al. Numerical simulating effects of rain-loaded wind on the aerodynamic characteristics and running stability of high-speed trains $[\mathrm{J}]$. Journal of Desert Research， 2016， 36(4): 943-950.

[8] 倪守隆. 风雨与沙尘环境下列车运行安全性研究 [D]. 大连: 大连交通大学, 2015 .

NI Shoulong. Study on the running safety of train under rain and sandstorm conditions[D]. Dalian: Dalian Jiaotong University, 2015.

[9] SHAO X M, WAN J, CHEN D W, et al. Aerodynamic modeling and stability analysis of a high-speed train under strong rain and crosswind conditions $[\mathrm{J}]$. Journal of
Zhejiang University - Science A (Applied Physics \& Engineering), 2011, 12(12): 964-970.

[10] 于梦阁, 李海庆, 刘加利, 等. 强风雨环境下高速列车 空气动力学性能研究 [J]. 机械工程学报, 2020, 56(4): 185-192.

YU Mengge, LI Haiqing, LIU Jiali, et al. Study on the aerodynamic performance of the high-speed train under strong wind and rainfall environment[J]. Jounrnal of Mechanical Engineering, 2020， 56(4): 185-192.

[11] WU Z L, CAO Y H. Numerical simulation of flow over an airfoil in heavy rain via a two-way coupled Eulerian-Lagrangian approach[J]. International Journal of Multiphase Flow, 2015，69: 81-92.

[12] MORSI S A, ALEXANDER A J. An investigation of particle trajectories in two-phase flow systems[J]. Journal of Fluid Mechanics, 1972, 55: 193-208.

[13] HAIDER A, LEVENSPIELl O. Drag coefficient and terminal velocity of spherical and nonspherical particles[J]. Powder Technology, 1989, 58(1): 63-70.

[14] BEST A C. The size distribution of raindrops[J]. Quarterly Journal of the Royal Meteorological Society, 1950, 76(32): $16-36$.

[15] MARKOWITZ A H. Raindrop size distribution expressions[J]. Journal of Applied Meteorology, 1976, 15: $1029-1031$

[16] 于梦阁, 刘加利, 李田. 基于非球形雨滴的强风雨环境 数值模拟 $[\mathrm{C}] / /$ 第十八届全国计算流体力学会议, 6 月 13 日-16日，西安，中国，2019。

YU Mengge, LIU Jiali, LI Tian. Numerical simulation of strong wind and rain environment based on non-spherical raindrops $[\mathrm{C}] / /$ The 18th National Conference on Computational Fluid Dynamics, June 13-16, Xi'an, China, 2019.

[17] 于梦阁, 张继业, 张卫华. 平地上高速列车的风致安全 特性[J]. 西南交通大学学报, 2011, 46(6): 989-995. YU Mengge , ZHANG Jiye , ZHANG Weihua. Wind-induced security of High-speed trains on the ground[J]. Journal of Southwest Jiaotong University, 2011, 46(6): 989-995.

[18] WANG X, QIAN Y H, CHEN Z S, et al. Numerical studies on aerodynamics of high-speed railway train subjected to strong crosswind[J]. Advances in Mechanical Engineering, 2019, 11(11): 1-12.

[19] 王政, 李田, 张继业. 不同类型横风下高速列车气动性 能研究[J]. 机械工程学报，2018，54(4)：203-211。

(下转第 193 页) 
presence of measurement faults[J]. ISA Transactions, 2010, 49(3): 249-256.

[35] HAJIYEV C, SOKEN H E. Robust adaptive unscented Kalman filter for attitude estimation of pico satellites[J]. International Journal of Adaptive Control and Signal Processing, 2014, 28(2): 107-120.

[36] RAHIMI A, KUMAR K D, ALIGHANBARI H. Fault estimation of satellite reaction wheels using covariance based adaptive unscented Kalman filter[J]. Acta astronautica, 2017, 134(5): 159-169.

[37] MENF Y, GAO S, ZHONG Y, et al. Covariance matching based adaptive unscented Kalman filter for direct filtering in INS/GNSS integration[J]. Acta Astronautica, 2016, 120: $171-181$.

作者简介: 张志达, 男, 1990 年出生, 博士研究生。主要研究方向为智 能汽车状态估计与循迹控制。

E-mail: zhangzhida_edu@163.com

郑玲(通信作者), 女, 1963 年出生, 博士, 教授, 博士研究生导师。主 要研究方向为智能汽车的环境感知、决策与动力学控制。

E-mail: zling@cqu.edu.cn

李以农, 男, 1961 年出生, 博士, 教授, 博士研究生导师。主要研究方 向为车辆系统动力学与控制、振动噪声控制。

E-mail: ynli@cqu.edu.cn

\section{(上接第 180 页)}

WANG Zheng, LI Tian, ZHANG Jiye. Research on aerodynamic performance of high-speed train subjected to different types of crosswind[J]. Jounrnal of Mechanical Engineering, 2018, 54(2): 203-211.

[20] CHOI E C C. Wind-drive rain and driving rain coefficient during thunderstorms and non-thunderstorms[J]. Journal of Wind Engineering and Industrial Aerodynamics, 2001, 89(3): 293-308.

[21] 张在中, 周丹. 不同头部外形高速列车气动性能风洞试 验研究[J]. 中南大学学报, 2013, 44(6): 2603-2608. ZHANG Zaizhong, ZHOU Dan. Wind tunnel experiment on aerodynamic characteristic of streamline head of high-speed train with different head shapes[J]. Journal of Central South University, 2013，44(6): 2603-2608.

[22] YU M G, JIANG R C, ZHANG Q, et al. Crosswind stability evaluation of high-speed train using different wind models $[\mathrm{J}]$. Chinese Journal of Mechanical Engineering, 2019, 32: 40.

[23] 于梦阁. 基于可靠性的高速列车风致安全研究 [D]. 成 都: 西南交通大学, 2014.

YU Mengge. Study on the wind-induced safety of the high-speed train based on the reliability[D]. Chengdu: Southwest Jiaotong University, 2014.

作者简介: 于梦阁, 女, 1985 年出生, 博士, 副教授。主要研究方向为 车辆空气动力学, 车辆系统动力学。

Email: yumengge0627@163.com

刘加利(通信作者), 男, 1985 年出生, 博士, 高级工程师。主要研究方 向为列车空气动力学。

Email: liujiali0612@163.com 